\title{
Dynamique De La Mangrove De Thiobon Dans \\ L'estuaire De La Casamance (Sénégal) \\ Entre 1972 Et 2017
}

\author{
Boubacar Solly \\ El Hadji Balla Dièye \\ Tidiane Sané
}

Département de Géographie, Laboratoire de Géomatique et d'Environnement, Université Assane Seck de Ziguinchor, Sénégal

\section{Amadou Tahirou Diaw}

Département de Géographie, Laboratoire d'Enseignement et de Recherche en Géomatique, Université Cheikh Anta Diop, Sénégal

Doi: 10.19044/esj.2018.v14n33p118 URL:http://dx.doi.org/10.19044/esj.2018.v14n33p118

\begin{abstract}
In the village soil of Thiobon in lower Casamance (southern Senegal), site being established as Marine Protected Area (MPA), the main activities of the population (rice growing, harvesting of fish products, salt production, ...), happens in mangrove zone. These mobilize the population for several months in the year. This mangrove ecosystem, vulnerable and fragile, has undergone profound changes since the 1960s under the combined actions of rainfull variability and main. This study proposes to analyze the spatio-temporal dynamics of Thiobon mangrove. The method used is based on the processing and analysis of satellite data (Landsat imagery acquired in 1972, 1986, 2000 and 2017), rainfall data (1960 to 2017), and on the perception of populations of change in their terroir (field investigation). Map results indicate an overall decline on 52\% in mangrove area in tanne during drought years (1970 to the late 1990s). During 2000-2017, characterized by the almost normal return of the rainfall and activities of reforestation done by the local population, about $69 \%$ of the mangrove areas lost between 1972 and 2000 regenerated. This dynamic is the result of external and internal forces exerted on this ecosystem and evolve since the early 1970s.
\end{abstract}

Keywords: Dynamic, Mangrove, Remote sensing, MPA, Thiobon 


\section{Résumé}

Dans le terroir villageois de Thiobon en Basse-Casamance (sud du Sénégal), site en cours d'être érigé en Aire Marine Protégée (AMP), les principales activités de la population (riziculture, prélèvement de produits halieutiques, saliculture, etc.) se déroulent en zone de mangrove. Celles-ci mobilisent la population pendant plusieurs mois dans l'année. Cet écosystème de mangrove, vulnérable et fragile, a connu de profondes mutations depuis les années 1960 sous les actions combinées de la variabilité pluviométrique et de l'homme. Cette étude se propose d'analyser la dynamique spatiale et temporelle de la mangrove de Thiobon. Elle se base sur le traitement et l'analyse des données satellitaires (images du satellite Landsat acquises en 1972, 1986, 2000 et 2017), des données pluviométriques (1960 à 2017) et sur la perception des populations des changements intervenus dans leur terroir (enquêtes sur le terrain). Les résultats cartographiques indiquent une régression globale de $52 \%$ des surfaces occupées par la mangrove au profit des tannes durant les années de sécheresse (de 1970 à la fin des années 1990). Durant la période 2000-2017 caractérisée par le retour quasi normal de la pluviométrie et la mise en œuvre des activités de reboisement de la mangrove effectuées par la population locale, environ $69 \%$ des surfaces de mangrove perdues entre 1972 et 2000 ont régénéré. Cette dynamique résulte des forces externes et internes qui s'exercent sur cet écosystème et le font évoluer depuis le début des années 1970.

Mots clés: Dynamique, Mangrove, Télédétection, AMP, Thiobon

\section{Introduction}

La mangrove désigne, entre autres, un arbre ou une formation végétale, un marais maritime tropical, un écosystème littoral forestier ou aquatique, un système à usage multiple, un paysage amphibie (Cormier-Salem, 1999). Les espèces végétales qui la composent sont désignées sous le terme de «palétuviers ». Au Sénégal, sept (7) espèces sont rencontrées (UNEP-WCMC, 2007) dont six (6) en Casamance (Marius, 1985) et cinq (5) à Thiobon (Solly, 2015). Il s'agit du Rhizophora mangle, du Rhizophora racemosa, du Rhizophora harrisonii, d'Avicennia germinans et de Laguncularia racemosa. En tant qu'écosystème, la mangrove est devenue l'objet d'un intérêt scientifique croissant lié à ses fonctions et à sa dynamique. Elle constitue un support à la biodiversité, un lieu d'habitat, de nichoir, de reproduction et de nourricerie pour de nombreuses espèces animales et végétales (CormierSalem, 1994 et 1999; Carrere et al., 2009). De même, elle régule le climat, protège les espaces habités contre les vagues et les vents violents, séquestre le 
carbone et fournit une gamme importante pour l'alimentation et la médecine traditionnelle (UNEP-WCMC, 2007). Toutefois, elle correspond à un milieu dynamique et fragile sous l'effet combiné de facteurs naturels et anthropiques (Moreau, 1991 ; Cormier-Salem, 1994 ; Andrieu, 2008 ; Dièye et al., 2013 ; Solly, 2015 ; Dièye et al., 2015). Ainsi, l'objectif de cette étude est d'analyser la dynamique spatio-temporelle de la mangrove de Thiobon en rapport avec l'évolution de la pluviométrie et les activités socio-économiques des populations. Ce site, en cours d'être érigé en Aire Marine Protégée (AMP), est de plus en plus le théâtre de recherches scientifiques et d'activités socioéconomiques.

\section{Présentation de la zone d'étude}

La zone d'étude est le terroir villageois de Thiobon. Elle est délimitée par les longitudes $16^{\circ} 59^{\prime}$ et $16^{\circ} 51^{\prime}$ 'Ouest et les latitudes $12^{\circ} 92^{\prime}$ et $12^{\circ} 83^{\prime}$ Nord. De son vrai nom Ekhimbane, Thiobon est situé en Basse-Casamance (Sud du Sénégal). Il est limité à l'Est par le village de Kartiack, à l'Ouest par le marigot 'Etoupaye', au Nord par le marigot de Baïla et au Sud par la commune de Mlomp (fig. 1). Il est à $42 \mathrm{~km}$ de la ville de Bignona, chef-lieu départemental et de $72 \mathrm{~km}$ de Ziguinchor, la capitale régionale.

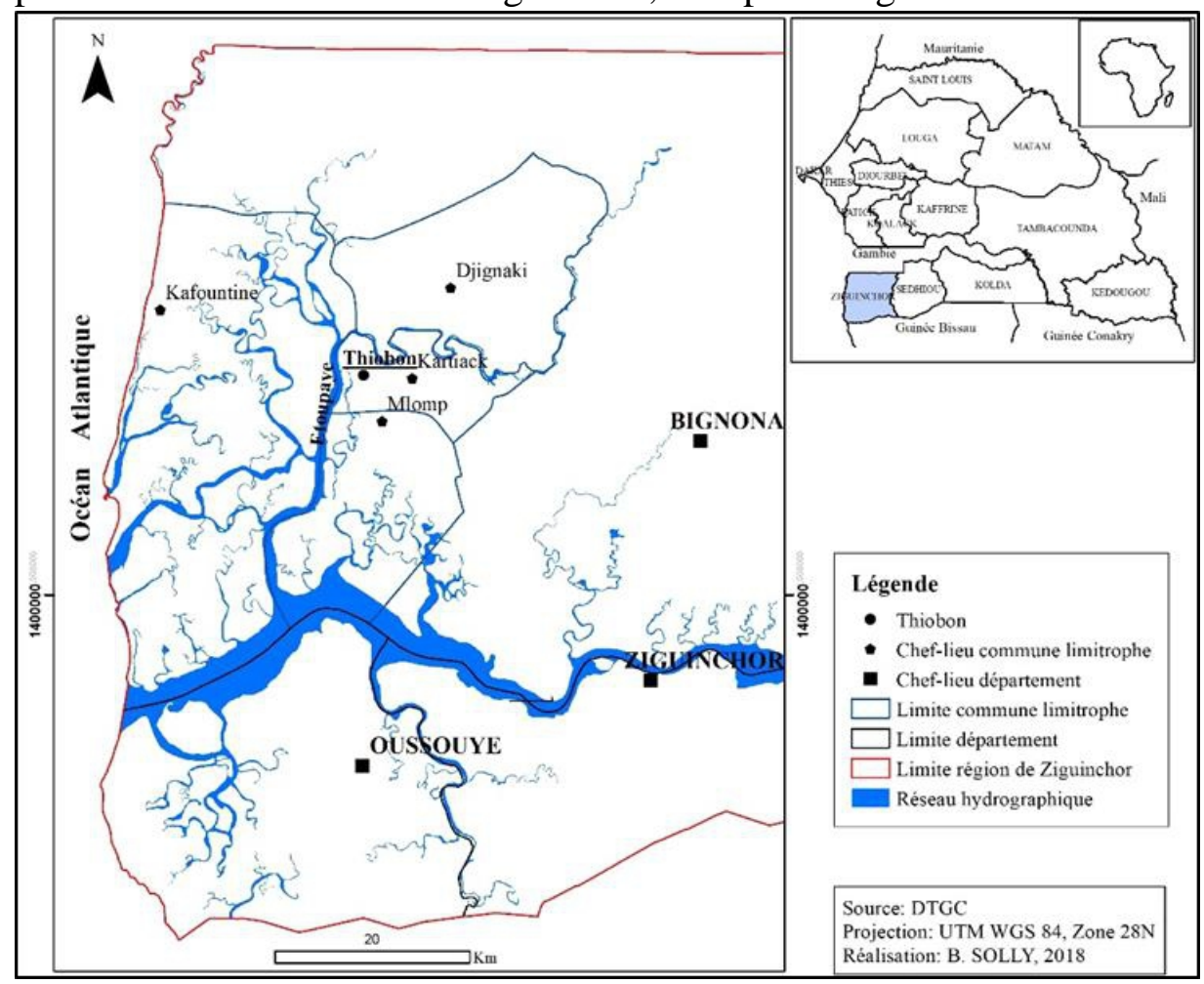

Figure 1 : Localisation du village de Thiobon en Basse-Casamance 
Elle bénéficie d'une pluviométrie relativement abondante qui dépasse en moyenne les $1000 \mathrm{~mm}$. Elle est située du point de vue climatique dans le domaine sud-soudanien côtier (Sagna, 2005). Elle présente deux saisons bien distinctes : une saison sèche caractérisée par la présence de l'alizé maritime qui dure en moyenne six à sept mois (novembre à mai) et une saison des pluies allant de juin à octobre influencée par l'arrivée de la mousson. La population est estimée à 2107 habitants en 2013 (RGPHAE, 2013).

\section{Donnees et Methodes}

\section{Traitement et analyse des images satellitaires}

\section{$\checkmark$ Données utilisées}

Pour la cartographie de l'évolution spatio-temporelle de la mangrove de Thiobon, nous avons utilisé les images du satellite Landsat. Ce dernier propose une couverture complète de la zone d'étude. Les dates de prise de vue des images sont 1972, 1986, 2000 et 2017. Les images sont produites dans des résolutions suffisantes pour la détection des changements intervenus dans la mangrove de Thiobon (tabl. 1). Pour éviter le bruit lié à la couverture nuageuse en saison des pluies, les images prises entre novembre et février (saison sèche) ont été retenues.

Tableau 1 : Images satellites Landsat utilisées

\begin{tabular}{|c|c|c|c|c|}
\hline Satellite & Série & Capteur & Date & Résolution spatiale \\
\hline \multirow{4}{*}{ Landsat } & L1 & MSS & $05-11-1972$ & $60 \mathrm{~m}$ \\
\cline { 2 - 5 } & L5 & TM & $09-02-1986$ & $30 \mathrm{~m}$ \\
\cline { 2 - 5 } & L7 & ETM+ & $06-11-2000$ & $30 \mathrm{~m}$ \\
\cline { 2 - 5 } & L8 & OLI_TIRS & $31-12-2017$ & $30 \mathrm{~m}$ \\
\hline
\end{tabular}

\section{$\checkmark$ Correction géométrique des images}

L'image Landsat acquise avec le capteur Multi Spectral Scanner en 1972 est à une résolution spatiale de $60 \mathrm{~m}$; alors que les autres images sont à une résolution de $30 \mathrm{~m}$. Elle a été ainsi rééchantillonnée à $30 \mathrm{~m}$ en utilisant la méthode du plus proche voisin qui utilise la valeur du pixel la plus proche, sans aucune interpolation, pour créer la valeur du pixel rectifié.

La correction géométrique a ensuite consisté, à ramener les images à la même géométrie. Pour superposer des images prises par des capteurs différents à des dates différentes, il est nécessaire qu'elles présentent le même géoréférencement (Masse, 2013 ; Andrieu, 2008). La méthode de correction image par image a été adoptée avec comme référence l'image de 2000 . La géométrie des images Landsat de 1972 (rééchantillonnée), de 1986 et de 2017 ont été corrigées en référence à Lourenco et al., (2009), avec un polynomial de degré $n$ dont le nombre de points de contrôle au sol ou GCP (Ground Control Points) sélectionné est supérieur à $(\mathrm{n}+1)^{2}$ et une erreur résiduelle 
inférieure à 1. Le logiciel de traitement d'image Envi 4.5 a été utilisé dans cette étape et pour la classification des images.

\section{$\checkmark$ Classification supervisée des images}

Le but de la classification supervisée est d'aboutir à des cartes thématiques. Le choix de cette méthode de classification est basé sur une bonne connaissance de la zone d'étude et la prise sur le terrain d'un nombre assez suffisant de points GPS. La méthode requiert de définir au préalable un nombre précis de classes thématiques. À Thiobon, nous avons identifié sur le terrain, six (6) classes thématiques réparties comme suit : eau, mangrove (palétuviers), tanne (surfaces recouvertes temporellement d'eau et/ou dénudées), rizière de mangrove (rizières situées à l'intérieur de la mangrove), rizière de plateau (rizières situées à l'interface entre la mangrove et la terre ferme), et autres substrats (autres végétations, habitats, sols nus) (fig. 2).

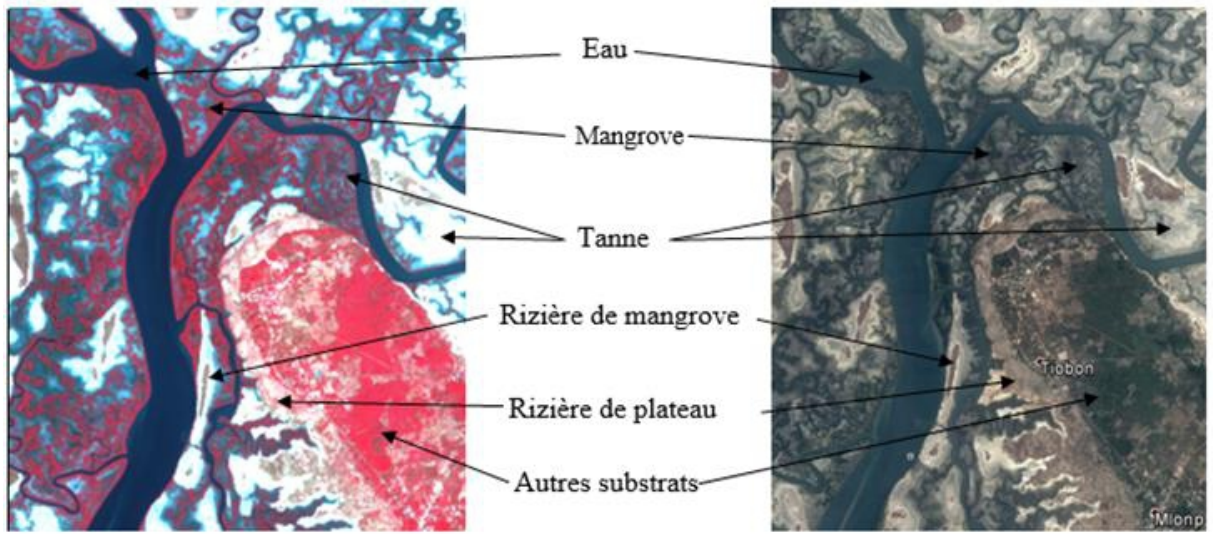

Figure 2 : Classes thématiques présentes dans la zone d'étude (source : image OLI_TIRS 2017 et Google Earth 2017)

À chacune de ces classes thématiques, un certain nombre de zones d'échantillonnage ou région d'intérêt $(R O I)$ sont définies par la numérisation de plusieurs polygones. L'algorithme maximum de vraisemblance est utilisé pour lancer la classification. Il s'agit de l'algorithme le plus utilisé pour la classification d'images en télédétection, car il est efficace et s'appuie sur des considérations théoriques approfondies et fournit de meilleurs résultats (Caloz et Collet, 2001). Il associe à la signature spectrale une distribution statistique connue et offre la possibilité d'affecter à chaque pixel une probabilité d'appartenance à une classe donnée (Girard et Girard, 2010 ; Masse, 2013).

\section{$\checkmark$ Vérification de la classification}

L'évaluation de la qualité des classifications est basée sur un minimum de 30 points de référence pris sur le terrain. L'interprétation visuelle des images à plus haute résolution (images Google Earth de 2017 et 2003) a été 
associée à notre connaissance du terrain pour évaluer la qualité des classifications. Aussi, l'indice de séparabilité Jeffries-Matusita a été calculé pour une meilleure discrimination des classes et produire une classification supervisée la plus précise possible. Le résultat des valeurs de séparabilité entre les classes et pour les différentes années est supérieur à 1,5. Ceci indique une bonne séparation des classes thématiques retenues (Vasconcelos et al., 2002).

\section{$\checkmark$ Cartographie diachronique}

Pour étudier les changements intervenus entre deux dates, une cartographie de l'occupation du sol a été effectuée sur la base de la classification des images pour chacune des années retenues. À la suite, un recodage des classes a été effectué. Un même code a été donné à chaque classe et sur toutes les images de sorte à pouvoir les croiser par addition des images (tabl. 2).

Tableau 2 : Codage des différentes classes

\begin{tabular}{|c|c|}
\hline Classe & Code \\
\hline Eau & 1 \\
\hline Mangrove & 2 \\
\hline Tanne & 3 \\
\hline Rizière de mangrove & 4 \\
\hline Rizière de plateau & 5 \\
\hline Autres substrats & 6 \\
\hline
\end{tabular}

À partir du recodage, trois situations sont possibles. Il s'agit de la régression (disparition), la progression (apparition) et la stabilité (sans changement). La régression indique une perte de superficie d'une classe d'une date à une autre. Autrement dit, il s'agit de la transformation d'une classe à une autre en termes de diminution de sa surface initiale. Les superficies perdues sont récupérées par les classes sous la forme de progression. Celle-ci correspond à l'augmentation de superficie d'une classe. La stabilité correspond à l'absence de changement. Le cumul de la stabilité et de la progression donne la superficie totale de la zone d'étude.

Pour étudier les changements, le logiciel de cartographie ArcGis 10.2 a été utilisé.

\section{Données pluviométriques et traitements}

Pour apprécier l'influence des précipitations sur l'évolution de la mangrove, le calcul des anomalies standardisées de la pluviométrie de la zone a été effectué. Thiobon étant dépourvu de station pluviométrique, les données de la station de Diouloulou, la plus proche, sont utilisées sur la période 19602017. 


\section{Enquêtes de terrain et traitement des données}

Des données qualitatives et quantitatives ont été collectées sur le terrain à travers l'administration d'un questionnaire et d'un guide d'entretien. Les informations recherchées ont porté essentiellement sur l'importance socio-économique et environnementale de la mangrove dans le terroir villageois de Thiobon. Il s'agit, entre autres, des différents types d'activités exercées dans la mangrove et leurs conséquences sur son évolution, de l'évolution du climat, principalement de la pluviométrie, et ses conséquences sur l'écosystème. La question des stratégies développées par la population pour la restauration et la sauvegarde de la mangrove a été également prise en compte.

Le questionnaire a été administré à 86 personnes, dont 50 chefs de ménages et 36 exploitants. La méthode d'échantillonnage de commodité a servi comme base d'échantillonnage. C'est une méthode qui consiste à prendre des individus suivant leur disponibilité pour participer à l'enquête. Selon Diatta et Diouf (2013), elle a l'avantage de donner de bons résultats quand la population est homogène. Pour compléter les informations collectées à partir du questionnaire, des entretiens ont été réalisés avec des personnes ressources. Il s'agit du président de l'Association pour la Rénovation de Thiobon (ASSORETH), du responsable des activités de reboisement de la mangrove, du président des pêcheurs, du président chargé de la protection de l'environnement et du chef de village. Le traitement des informations quantitatives collectées s'est fait de manière automatique et par des tris à plat à partir du logiciel Sphinx Plus.

\section{Résultats}

\section{Cartographie de l'évolution spatio-temporelle de la mangrove de Thiobon entre 1972 et 2017}

La figure 3 montre les changements intervenus dans l'écosystème de mangrove de Thiobon au cours des périodes 1972-1986, 1986-2000 et 20002017. Elle indique l'importance de la mangrove disparue, de la mangrove stable et des tannes entre 1972-1986. Il s'agit, en effet, d'une conversion de certaines surfaces de mangrove en surfaces de tannes. Entre 1986-2000, la figure indique l'importance des tannes et la stabilité de la mangrove. Entre 2000-2017, la figure fait état de l'importance de la mangrove apparue et une reconversion de certaines surfaces de tannes en surfaces de mangrove. 


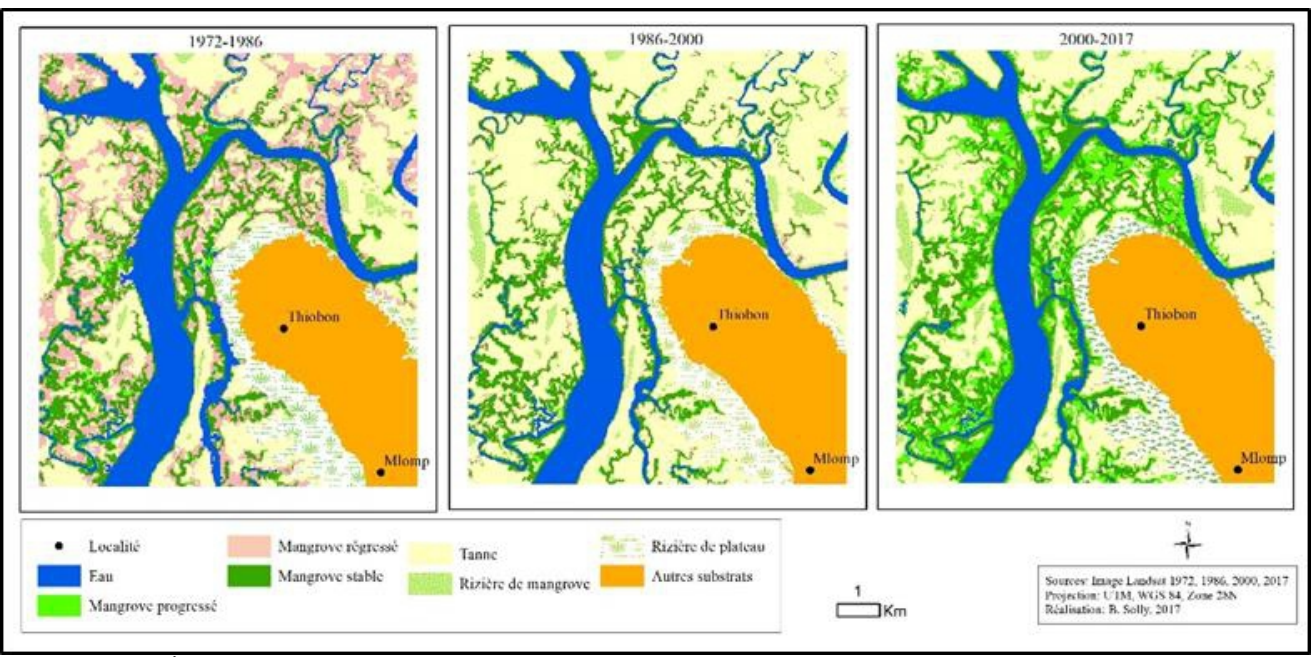

Figure 3 : Évolution de la mangrove de Thiobon entre 1972-1986, 1986-2000 et 2000-2017

Le tableau 3 montre les changements notés entre 1972 et 1986. Il indique une perte importante des surfaces de mangrove. Au total, 1482 ha de mangrove ont disparu. Cette réduction de la mangrove s'est accompagnée d'une augmentation des tannes de 1613,8 ha. Les surfaces occupées par l'eau, les rizières de mangrove et autres substrats ont évolué respectivement de 96,6 ha, 14,7 ha et 84,6 ha. Les rizières de plateau par contre ont connu une perte de 328,1 ha.

Tableau 3 : Changements intervenus entre 1972 et 1986 en hectare

\begin{tabular}{|c|c|c|c|c|}
\hline Classes & Stable & Progression & Régression & $\begin{array}{c}\text { Gain (+) ou } \\
\text { Perte (-) de } \\
\text { surface }\end{array}$ \\
\hline $\mathrm{Eau}$ & 1058,5 & 216 & 119,1 & $+96,9$ \\
\hline Mangrove & 1130,3 & 251 & 1733 & -1482 \\
\hline Tanne & 2135,4 & 1888,6 & 274,8 & $+1613,8$ \\
\hline Rizière de mangrove & 42,4 & 42 & 27,3 & $+14,7$ \\
\hline Rizière de plateau & 192,1 & 42,5 & 370,6 & $-328,1$ \\
\hline Autres substrats & 1372,1 & 96,8 & 12,1 & $+84,6$ \\
\hline Total & \multicolumn{2}{|c|}{8467,7} & 2536,9 & \\
\hline
\end{tabular}

Le tableau 4 montre les changements intervenus entre 1986 et 2000. Comparée à la période 1972-1986, une faible régression de la mangrove a été notée. La mangrove a perdu 29,6 ha de sa superficie durant cette période. Les tannes ont perdu 98,4 ha. Les rizières de mangrove et de plateau ont connu respectivement une progression de 39,7 ha et 212,7 ha. L'eau a connu une perte de 127,9 ha. Cette période est marquée globalement par une stabilité des classes. 
Tableau 4 : Changements intervenus entre 1986 et 2000 en hectare

\begin{tabular}{|c|c|c|c|c|}
\hline Classes & Stable & Progression & Régression & $\begin{array}{c}\text { Gain (+) ou } \\
\text { Perte (-) de } \\
\text { surface }\end{array}$ \\
\hline Eau & 1116,5 & 21,8 & 149,7 & $-127,9$ \\
\hline Mangrove & 1062,4 & 306,4 & 336 & $-29,6$ \\
\hline Tanne & 3523,1 & 401,2 & 499,6 & $-98,4$ \\
\hline Rizière de mangrove & 79,3 & 47,3 & 7,6 & $+39,7$ \\
\hline Rizière de plateau & 143,0 & 296 & 83,3 & $+212,7$ \\
\hline Autres substrats & 1418,3 & 52,6 & 49 & $+3,6$ \\
\hline Total & \multicolumn{2}{|c|}{8467,7} & 1125,2 & \\
\hline
\end{tabular}

Le tableau 5 montre les changements intervenus durant la période 2000-2017. Contrairement aux tendances notées durant les deux premières périodes, la mangrove a connu une évolution positive durant la période 2000-2017. Elle a connu une progression de 1046,2 ha. Comparé à la situation de 1972-1986 et 1986-2000, environ $69 \%$ des surfaces disparues ont régénéré. Concomitamment à la régénération de la mangrove, les tannes ont connu une perte importante de 1020,3 ha. Ce changement peut s'expliquer par une prise de conscience des populations marquée par les reboisements effectués entre 2006 et 2016. Durant cette courte période, environ 1418 ha de palétuviers ont été reboisés. Cela a contribué sensiblement à l'augmentation des surfaces de mangrove ces dernières années. Les rizières de mangrove quant à elles ont connu une timide augmentation, et les rizières de plateau, une timide diminution.

Tableau 5 : Changements intervenus entre 2000 et 2017 en hectare

\begin{tabular}{|c|c|c|c|c|}
\hline Classes & Stable & Progression & Régression & $\begin{array}{c}\text { Gain (+) ou } \\
\text { Perte (-) de } \\
\text { surface }\end{array}$ \\
\hline Eau & 1098,9 & 44,5 & 39,3 & $+5,1$ \\
\hline Mangrove & 1310,4 & 1104,6 & 58,4 & $+1046,2$ \\
\hline Tanne & 2803,9 & 100,1 & 1120,4 & $-1020,3$ \\
\hline Rizière de mangrove & 110,5 & 18 & 16 & +2 \\
\hline Rizière de plateau & 365,9 & 53,3 & 73 & $-19,7$ \\
\hline Autres substrats & 1437 & 20,6 & 33,8 & $-13,2$ \\
\hline Total & \multicolumn{2}{|c|}{8467,7} & 1341 & \\
\hline
\end{tabular}

\section{Evolution de la pluviométrie et son influence sur la dynamique de la mangrove de Thiobon}

De nombreux auteurs ont montré la corrélation qui existe entre l'évolution de la mangrove et celle de la pluviométrie (Marius, 1979; Moreau, 1991 ; Andrieu, 2008; Sy et Dieng, 2009 ; Dièye et al., 2013). En effet, la variabilité de la pluie influence nettement l'évolution de la mangrove. Or, une variabilité pluviométrique marquée par un important déficit a été notée dans la zone de 1968 à la fin des années 1990 (Bassel, 1993 ; Sagna, 2005 ; Sané et 
al., 2013; Solly, 2015). L'analyse des anomalies standardisées de la pluviométrie annuelle de Diouloulou (fig. 4) fait ressortir trois grandes périodes : 1960-1967, 1968-1998 et 1999-2017.

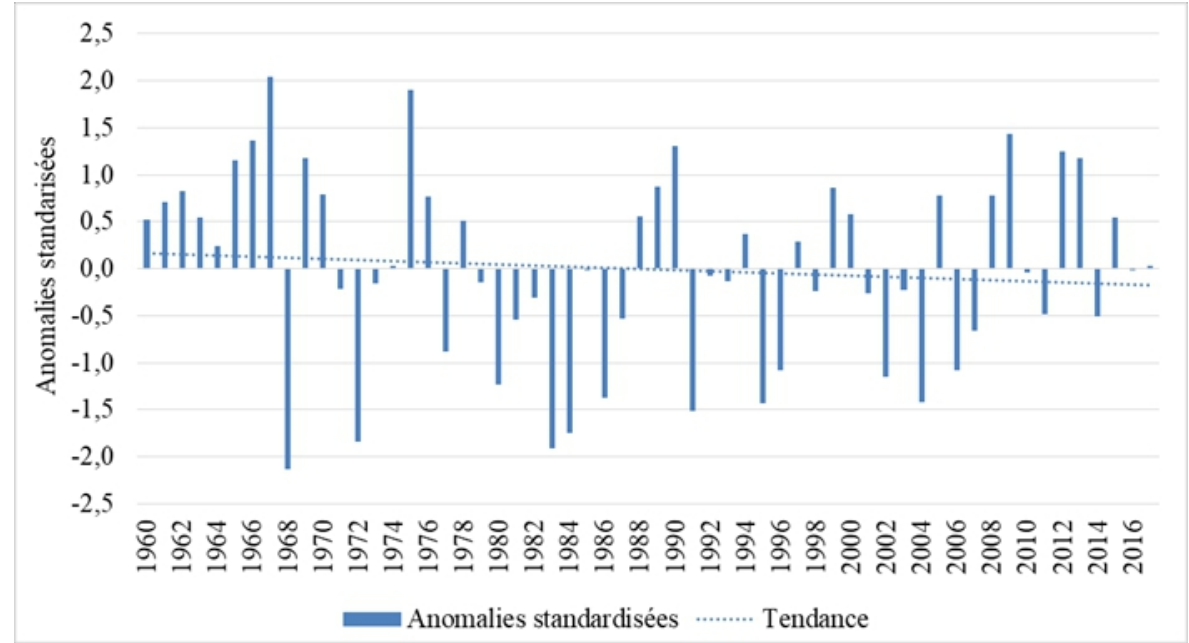

Figure 4 : Évolution des anomalies standardisées de la pluviométrie à Diouloulou (1960 à 2017)

La période 1960-1968 est caractérisée par des anomalies positives avec des indices pluviométriques relativement importants qui varient entre 0,2 et 2 . Ces indices révèlent le caractère relativement humide de cette période. Les conditions pluviométriques sont supérieures à la moyenne de la série qui est de $1109,9 \mathrm{~mm}$.

La période 1968-1998 est, par contre, marquée par la prédominance des anomalies négatives qui illustrent la sécheresse de cette période. Sur 31 ans, seules quelques rares années ont enregistré des indices pluviométriques légèrement positifs puisqu'elles dépassent rarement 0,1 . C'est le cas des années 1969-1970, 1975-1976, 1978, 1988-1990, 1994 et 1997. Selon les personnes interrogées, la baisse de la pluie a eu des effets négatifs sur la mangrove durant cette période. Elles estiment que cette sécheresse a favorisé l'augmentation des tannes, le stress hydrique des plantes avec comme conséquence la mort des palétuviers par carence en oxygène $\left(\mathrm{O}_{2}\right)$ notamment le genre Rhizophora qui supporte mal des quantités trop élevées de sel, et la salinisation des sols.

Contrairement à ces deux périodes, celle de 1999-2017 est caractérisée par une alternance entre années pluvieuses et celles non pluvieuses. Les indices pluviométriques ont varié entre $-1,4$ et 1,4 durant cette période avec une nette amélioration de la moyenne pluviométrique qui est passée de $1099,3 \mathrm{~mm}$ à 1131,9 mm. Le caractère relativement pluvieux de cette période et les activités de reboisement de la mangrove ont contribué à l'augmentation des surfaces de mangrove durant ces dernières années. 


\section{Activités d'exploitation et de revalorisation et leur influence sur la dynamique de la mangrove de Thiobon}

À Thiobon, la mangrove revêt une importance socio-économique et environnementale certaine pour la population locale. Compte tenu de son potentiel en ressources naturelles, différentes activités d'exploitation sont pratiquées dans l'écosystème de mangrove. Il s'agit principalement de la coupe du bois, de la cueillette des huîtres, de l'extraction de sel, de la pêche et du fumage de poissons (fig. 5).

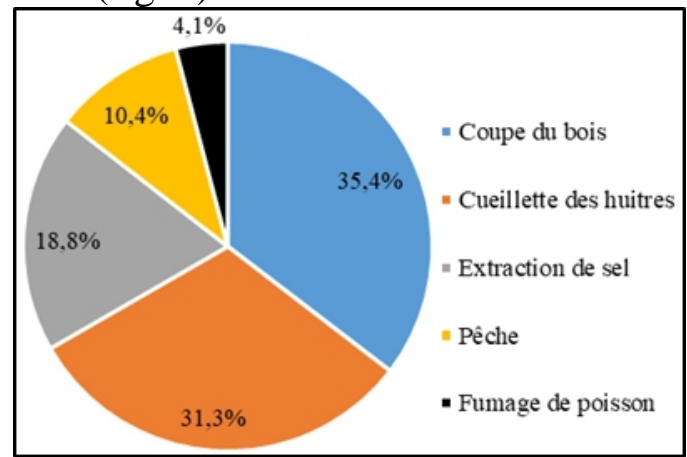

Figure 5 : Principales activités d'exploitation dans la mangrove de Thiobon

Ces activités contribuent aux bonnes conditions de vie des populations et leur procurent des revenus. Parmi ces activités, deux principalement selon la population, ont influencé l'évolution de la mangrove. Il s'agit de la coupe du bois et de la cueillette des huîtres.

La coupe du bois est exercée par 35,4\% des personnes interrogées. Pour la cuisine, la cuisson des huîtres, le fumage, la clôture, le jardinage, le plafonnage, etc., la population n'utilisent pratiquement que le bois de mangrove (fig. 6).
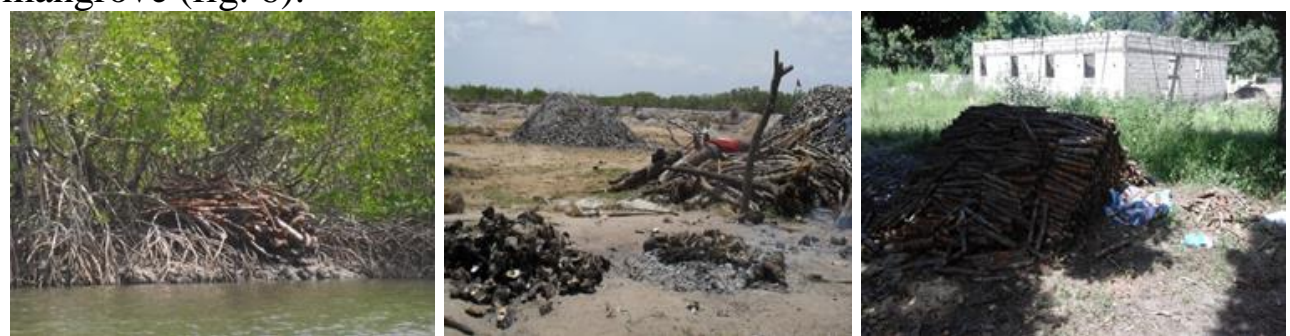

Figure 6 : Quelques exemples de coupe et d'utilisation du bois de mangrove à Thiobon

Le bois sec est régulièrement utilisé par $65,5 \%$ de la population enquêtée; contre 34,5\% qui utilisent le bois humide. Pour la cuisine, la cuisson des huîtres et le fumage, le bois de Rhizophora est le plus utilisé. Il est très commode et résistant, car il s'allume facilement même durant la saison des pluies et engendre moins de fumée comparée au bois d'Avicennia. Toutefois, selon certaines personnes enquêtées, ce bois procure beaucoup plus 
de charbon une fois allumé. De même, le bois de Rhizophora est utilisé pour la clôture et le jardinage. Les racines échasses sont utilisées pour le plafonnage. En plus de son utilisation domestique, le bois de mangrove est commercialisé dans le passé. Même si cette pratique semble disparue, les espèces coupées mesurent 3 à $5 \mathrm{~m}$ de hauteur et de manière incontrôlée.

Quant à la cueillette des huîtres, elle demeure une activité traditionnelle des femmes diola en Basse-Casamance particulièrement à Thiobon où elle génère des revenus annuels qui varient entre 150000 F CFA et $200000 \mathrm{~F}$ CFA par exploitante. Elle est pratiquée par $31,3 \%$ des exploitantes, parmi lesquelles, certaines avaient tendance à couper les racines échasses des palétuviers.

Parallèlement aux activités d'exploitation, la population a développé des activités de revalorisation basées sur la sensibilisation, le reboisement, et l'interdiction de coupe du bois humide. Ces actions, particulièrement le reboisement, ont contribué à l'augmentation sensible de la mangrove dans le terroir villageois de Thiobon. Ceci se confirme sur la carte d'évolution pour la période 2000-2017.

\section{Discussion}

La mangrove de Thiobon a connu une dynamique sous l'influence de la variabilité pluviométrique, de la coupe du bois (bois de chauffe et de service), de l'activité de cueillette, et des actions de réhabilitation de l'écosystème. Les résultats de cette étude concordent avec les tendances notées dans le delta du Saloum (Dièye, 2007; Andrieu, 2008), et celles observées dans l'estuaire de la Casamance (Moreau, 1991; Conchedda et al., 2007 ; Sy et Dieng, 2009; Dièye et al., 2013). Les résultats révèlent trois scénarios. Le premier correspond à la perte de la mangrove (1482 ha), l'augmentation des tannes (1613,8 ha), la baisse de la productivité et la disparition et/ou rareté de certaines espèces animales entre 1972 et 1986. Cette situation s'explique principalement par la baisse de la pluviométrie notée durant cette période, autrement dit, par la sécheresse des années 1970 et 1980 (Marius, 1979 ; Moreau, 1991). Elle s'explique aussi par l'augmentation de la teneur en sel qui empêche le renouvellement de la mangrove (Bassel, 1993). Cette longue période de sécheresse a entraîné, selon la population, une baisse importante de la productivité et l'exacerbation de la salinité des terres rizicoles devenues impropres à la riziculture. Cette situation a favorisé chez les populations locales la mise en œuvre de stratégies de survie. C'est ainsi que certains chefs de ménages se sont déployés dans la coupe et la commercialisation du bois, et dans la cueillette des huîtres. Cette réorientation des activités n'a pas été favorable aux palétuviers. En effet, pour la construction des toits de maison, la population avait tendance à utiliser les racines échasses du Rhizophora en raison de la qualité du bois. Pour la 
cueillette des huîtres, les femmes coupaient les racines échasses. Pour la clôture et la cuisine, le bois de mangrove, particulièrement le Rhizophora était le seul utilisé durant cette période. Il est d'ailleurs couramment utilisé dans les pays tropicaux pour la cuisine, en raison de son pouvoir calorifique très élevé (ISME, 1995). Cette dégradation de la mangrove s'est traduite sur la faune par la disparition et/ou la rareté de certaines espèces, dont les crocodiles, les biches, les dauphins, les hippopotames et certaines espèces d'oiseaux migrateurs.

Le second scénario est relatif à une timide régression de la mangrove durant la période 1986-2000. La mangrove a très peu régressé durant cette période. Il faut souligner que cette période est aussi marquée par quelques rares années à pluviométrie normales à excédentaires (1988-1990, 1994 et 1997). Cette période qui fait partie de la longue période de sécheresse notée depuis la fin des années 60 a contribué à atténuer la régression de la mangrove.

Le dernier scénario est caractérisé par une augmentation des surfaces de mangrove, une diminution des surfaces occupées par les tannes, et une timide augmentation de la productivité. En effet, entre 2000 et 2017, la mangrove a connu une évolution d'environ 1046,2 ha. Les tannes ont diminué de 1020,3 ha. Ceci peut s'expliquer par le retour relatif de la pluviométrie favorable au développement des palétuviers (régénération naturelle) et par les activités de reboisement de la mangrove introduites depuis 2006. En effet, depuis 2006, la population, à travers l'ASSORETH, et avec l'appui des partenaires techniques et financiers (Oceanium et Danone) et le service technique de l'État (Service des Eaux et Forêts), a mis en place des stratégies de gestion de la mangrove basées sur la sensibilisation, le reboisement et la mise en place de dispositif de réglementation pour une meilleure gestion de la mangrove du terroir. Ce dispositif porte sur la coupe et les méthodes de cueillette des huîtres.

Ces stratégies ont permis une prise de conscience de la population sur l'importance de l'écosystème de mangrove. Cette prise de conscience s'est traduite entre autres par la signature d'un protocole d'accord entre la population de Thiobon et l'ONG Oceanium. Ce protocole porte sur l'interdiction de couper le bois dans les sites reboisés d'ici au moins 10 ans. Ainsi, l'augmentation de la superficie de la mangrove a contribué à l'introduction de nouvelles activités dans l'écosystème à l'image de l'apiculture et de l'ostréiculture en 2015.

Toutefois, malgré l'importance des surfaces de mangrove (2356,6 ha), les tannes restent toujours importantes (2803,9 ha) et pourraient s'expliquer par l'augmentation de la salinité notée à partir de 1988 (Bassel, 1993). En effet, cette salinité a entraîné une augmentation considérable des surfaces hyper-salées et stériles (tannes vifs) développées aux dépens de la mangrove (Loyer et al., 1986 ; Bassel, 1993). Selon Cormier-Salem (1994, 1999), cette 
situation est la même dans les autres pays des rivières du sud, car la baisse de la pluviométrie entraîne des modifications majeures de l'environnement et permet aux tannes de se développer dans des zones où se manifeste la sécheresse, mais aussi où la fréquence de submersion et le niveau de l'eau sont devenus faibles.

\section{Conclusion}

Le traitement et l'analyse des images satellitaires, des données pluviométriques et d'enquêtes de terrain ont permis de montrer la dynamique de l'écosystème de mangrove de Thiobon caractérisée par trois phases d'évolution. La première phase, de 1972 à 1986, est caractérisée par une diminution importante des surfaces de mangrove. Elle s'explique par la diminution drastique de la pluviométrie et les coupes répétées du bois de mangrove pour le chauffage et le service. La seconde phase qui va de 1986 à 2000 est caractérisée par une timide diminution de la mangrove. La dernière phase allant de 2000 à 2017 est marquée quant à elle, par une augmentation des surfaces de mangrove et une diminution des surfaces de tannes grâce, d'une part, à l'amélioration des conditions pluviométriques et, d'autre part, aux actions de restauration et de revalorisation développées par la population locale. La pérennisation de la dynamique actuelle et les initiatives locales de gestion et de sauvegarde de cet écosystème s'inscrivent bien dans la création d'une Aire Marine Protégée.

\section{References:}

1. Andrieu J. (2008). Dynamique des paysages dans les régions septentrionales des Rivières-du-Sud (Sénégal, Gambie, GuinéeBissau), Thèse de Doctorat, EESC, 532 p.

2. RGPHAE (2013). Recensement Général de la Population et de l'Habitat, de l'Agriculture et de l'Elevage de 2013. Rapport final, 417 p.

3. Bassel M. (1993). Conséquence des deux décennies de sécheresse, d'hypersalinisation de la Casamance de 1987 à 1992, Rapport de stage de D. E.A, Département de Géographie, UCAD, 23 p.

4. Caloz R., et Collet C. (2001). Précis de télédétection : Traitements numériques d'images de télédétection, volume 3 , presse de l'Université de Québec, 386 p.

5. Carrere R. (2009). Les mangroves africaines : leur importance pour les populations et la biodiversité, Nature et faune, vol 24, No1, 3-7.

6. Conchedda G., Durieux L., et Mayaux P. (2007). Object-based monitoring of land cover changes in mangrove ecosystems of Senegal, In : Analysis of Multi-temporal Remote Sensing Images, International Workshop on the. Leuven : IEEE, 6p. 
7. Cormier-Salem M. C. (1999). Rivière du sud: Société et mangrove ouest-africaine, Paris, édition IRD, vol 1, 416 p.

8. Cormier-Salem M. C. (1994). Dynamique et usage de la mangrove dans les pays des rivières du sud (du Sénégal à la Sierra-Léone), Paris, édition ORSTOM, 353 p.

9. Diatta A. J., et Diouf D. A. (2013). Le Mémoire : Méthodologie de recherche, normes et techniques de rédaction, conseils pour la soutenance, Ziguinchor, Imprimerie Néma, 101 p.

10. Dièye E.H.B., Sané T., Ndour N., Sy O., Ba B.D., Solly B., Tall E.S.B., et Mendy V. (2015). Dynamique de la mangrove et impacts dans le Département d'Oussouye (Basse Casamance) entre 1972 et 2014. Rapport projet BM-UASZ, SPF-C1DC, 41 .

11. Dièye E.H.B., Sané T., Manga A., Diaw A.T., et Diop M. (2013). Variabilité pluviométrique et dégradation des écosystèmes de mangrove: actions communautaires de réhabilitation à Tobor en Basse-Casamance, XXVIe colloque de l'Association Internationale de Climatologie, 194-199.

12. Dièye E.H.B. (2007). Les ensembles littoraux de la lagune de JoalFadiouth et de l'estuaire du Saloum (Sénégal): Approche méthodologique de la dynamique de la mangrove entre 1972 et 2005 par télédétection et système d'information géographique, Doctorant $3^{\mathrm{e}}$ cycle, FST/UCAD, LERG, Dakar, 217 p.

13. Girard M. C., et Girard C.M. (2010). Traitement des données de télédétection : Environnement et ressources naturelles, Paris, Dunod, 2e ed, 1999, 2004, 2010, 553 p.

14. ISME. (1995). Faites connaissance avec la mangrove, COMAR/ UNESCO, $32 \mathrm{p}$.

15. Lourenço P., Cabral A.I. R., Oom D., Vasconcelos M.J.P., Catarino L., Temudo M.P. (2009). Re-growth of mangrove forests of GuineaBissau, 3rd Intern. Symp. Remote Sensing of Environment, 4p.

16. Loyer J.Y., Bovin P., Le Brusq J.Y., et Zante P. (1986). Les sols du domaine fluviomarin de Casamance (Sénégal) : Évolution récente et réévaluation des contraintes majeures pour leur mise en valeur, Selected Papers of the Dakar Symposium on Acid Sulphate Soils, Dakar, Edited by H. Dost, 16-23.

17. Marius C. (1985). Mangroves du Sénégal et de la Gambie : écologie, pédologie, géochimie, mise en valeur et aménagement, Paris, ORSTOM, coll. Travaux et Documents, 357 p.

18. Marius C. (1979). Effets de la sécheresse sur l'évolution phytogéographique et pédologique de la mangrove en BasseCasamance, Bulletin de l'IFAN, Tome 41, Série. À, nº 4, 671-691. 
19. Masse A. (2013). Développement et automatisation de méthodes de classification à partir de séries temporelles d'images de télédétection : Application aux changements d'occupation des sols et à l'estimation du bilan carbone, Thèse de Doctorat en Traitement d'image appliqué à la télédétection, Université Toulouse III Paul Sabatier, 223 p.

20. Moreau N. (1991). Contribution de la télédétection à l'étude de l'évolution des paysages de mangroves de l'Afrique de l'ouest, Thèse de Doctorat, Université Michel de Montaigne bordeaux III, 272 p.

21. Sagna P. (2005). Dynamique du climat et son évolution récente dans la partie ouest de l'Afrique occidental. Thèse de Doctorat d'Eta Es Lettres, Université Cheikh Anta Diop, Département de Géographie, Tome 2, 271-786.

22. Sané T., Sow B.A., Dièye E.H.B., Camara M., et Diatta S. (2013). Impacts de la température de surface de la mer et du flux de mousson sur la pluviométrie en Basse-Casamance (sud-ouest du Sénégal), XXVIe colloque de l'Association Internationale de Climatologie, 452457.

23. Solly B. (2015). La mangrove de Thiobon en Basse-Casamance (Sénégal) : dynamique spatiale, impacts et stratégies de gestion. Mémoire de master, Département de Géographie, Université Assane Seck de Ziguinchor, $92 \mathrm{p}$.

24. Sy B.A., Dieng S.D. (2009). Etude de la dynamique actuelle de la mangrove d'Oukout en Basse-Casamance au Sénégal, In Revue de Géographie du Laboratoire Leïdi (RGLL), Université Gaston Berger, Sénégal, 23-44.

25. UNEP-WCMC. (2007). Mangroves of western and central Africa, Report produced for UNEP-DEPI under the UNEP Biodiversity Related Projects in Africa, $92 \mathrm{p}$.

26. Vasconcelos M.J.P., Mussa Biai J.C., Araujo A., Diniz M.A. (2002). Land cover change in two protected areas of Guinea-Bissau (19561998), Applied Geography 22, 139-156. 